\title{
Analysis of Vegetation Infection Information Using Unmanned Aerial Vehicle with Optical Sensor
}

\author{
Kap Yong Jung ${ }^{1}$ and Joon Kyu Park ${ }^{2 *}$ \\ ${ }^{1}$ Depatrment of Civil Engineering, Chungnam National University, \\ 99, Daehak-ro, Yuseong-gu, Daejeon 34134, Korea \\ ${ }^{2}$ Department of Civil Engineering, Seoil University, \\ 28, Yongmasan-ro 90-gil, Jungnang-gu, Seoul 02192, Korea
}

(Received June 2, 2019; accepted August 9, 2019)

Keywords: UAV, optical sensor, big data, ortho image, near-infrared image, infection information, forest management, Bursaphelenchus xylophilus

The forests (approx. 640000 ha) of Korea comprise coniferous forest (41\%), broad-leaved forest (27\%), and mixed stand forest (29\%). They appear to be vulnerable to fire, diseases, and pests. The pine tree is one of the typical Korean species of trees. It was more than $50 \%$ of the whole forest area of the country in the $1960 \mathrm{~s}$, but the area of pine tree forests has been reduced to $30 \%$ because of recent changes in the forest ecosystem and damage caused by diseases and insect pests. In particular, pine wilt disease is currently spreading over Korea. In this study, an unmanned aerial vehicle (UAV) with an optical sensor was used to detect infected trees and to support big data on forest management. Red, green, and blue (RGB) images and near-infrared (NIR) images were acquired using UAV. The infected trees were detected using the RGB images, and the normalized difference vegetation index (NDVI) values were calculated using NIR images. The NDVIs of infected trees were lower than those of non-infected ones, and infected trees that were not detected as infected ones in the RGB images also have lower NDVIs than the neighboring trees that were detected as being infected. Through further research, if a distinct feature of the NDVI of infected trees is discovered, it will be helpful for the early detection of infected trees. Hence, this research is expected to be applied to the detection of infected trees and to support big data on forest management.

\section{Introduction}

In today's data-driven society, we have been hearing a great deal about the power of big data over the last few years. Big data has wide applications ranging from situation awareness and decision-making on an area to enable a foreseeable future and analysis of data. ${ }^{(1,2)}$ It is necessary to build big data for the management of disasters. In this study, we utilized an unmanned aerial vehicle (UAV) for this purpose, focusing on Bursaphelenchus xylophilus (BURSXY).

*Corresponding author: e-mail: jkpark@seoil.ac.kr https://doi.org/10.18494/SAM.2019.2465 
BURSXY was first described in, and is perhaps native to, North America. The nematode occurs in 36 states in the U.S. including all the Great Plains states with the exception of North Dakota, as well as almost all prefectures in Japan and several provinces in China. ${ }^{(3,4)}$ It also occurs in Canada, Mexico, Taiwan, Korea, and Portugal. Several species of nematodes including BURSXY have a phoretic relationship with cerambycid longhorn beetles, also known as sawyers, which function as vectors and carry them to recently felled logs and dead or dying conifers, particularly pines. Longhorn beetles, nematodes, or both may be found in pine chips, unseasoned lumber, and logs. Consequently, they are easily transported in wood products, such as logs, lumber, pallets, crates, wood chips, and furniture, which are not kiln-dried. ${ }^{(5,6)}$

In 2011, the area damaged by BURSXY was about 5000 ha, and Korea spent 22200 million won on pest control measures. The forests of Korea comprise coniferous forest (41\%), broadleaved forest (27\%), and mixed stand forest (29\%). ${ }^{(7,8)}$ They are vulnerable to fire, diseases, and pests. Recently, in Jeju Special Self-Governing Province and Gyeongsangbuk-do, the widespread distribution of BURSXY has caused much damage. Figure 1 shows the current status of damage caused by BURSXY. ${ }^{(9)}$

The chemical and physical pest control in the damaged area proceeded after determining the current status on the basis of BURSXY's physiological characteristics. ${ }^{(10)}$ However, even from the stage of determining the current status of infected trees, existing field investigation methods show limits because of the poor accessibility of affected areas owing to not only the difficult terrain conditions but also the vastness of the area. Therefore, the current status of infected trees should be determined urgently by scientific and objective methods. Moreover, through periodic monitoring of the progress of pest control, an objective evaluation of additional occurrences and the effect of the pest control is needed. That is, for a damaged area, first, the

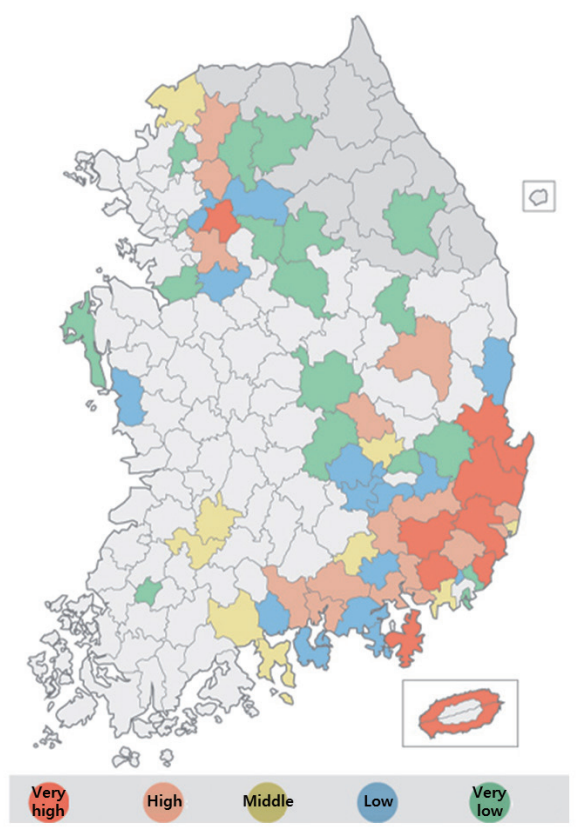

Fig. 1. (Color online) Current status of damage caused by BURSXY. 
current status should be determined by accurate and objective methods, and the area subjected to pest control should be monitored periodically. Furthermore, on the basis of red, green, and blue (RGB) images as well as near-infrared (NIR) images, BURSXY infection can be detected in the early stage and treated before the damage becomes widespread and extensive. ${ }^{(11)}$ In the literature on the application of aerial photography, the necessity of NIR was suggested many times, but practical research studies were poorly conducted.

In this study, an UAV was used to take images of the target area, and the images were processed as RGB ortho images and NIR images. We tried to detect the trees infected with BURSXY by effectively using the images and determined the applicability of NIR images for early detection. Figure 2 shows the study flow.

\section{Study Area and Image Acquisition}

The study area of this research was Ongcheon-ri, Andong-si (Fig. 3). Pest control was already carried out in this area, but the area still required the monitoring for newly infected trees; thus, it was chosen as the study area.

In this study, UX5 with optical sensors (RGB and NIR sensors) was used to obtain images for the analysis of the vegetation infection information. UX5 was equipped with a GPS, a digital camera, a radio antenna, a pitot tube, and other sensors. The GPS receiver measured the time difference of signals from each satellite and converted it into distance to estimate the location of the receiver. A Sony NEX-5R digital camera was used to take high-resolution pictures of the ground. The camera has a larger sensor than those of other companies' products of the same size, which could take high-quality pictures. Sony NEX-5R was equipped with an advanced photo system (APS)-C-type sensor similarly to a DSLR camera, and the APS-C-

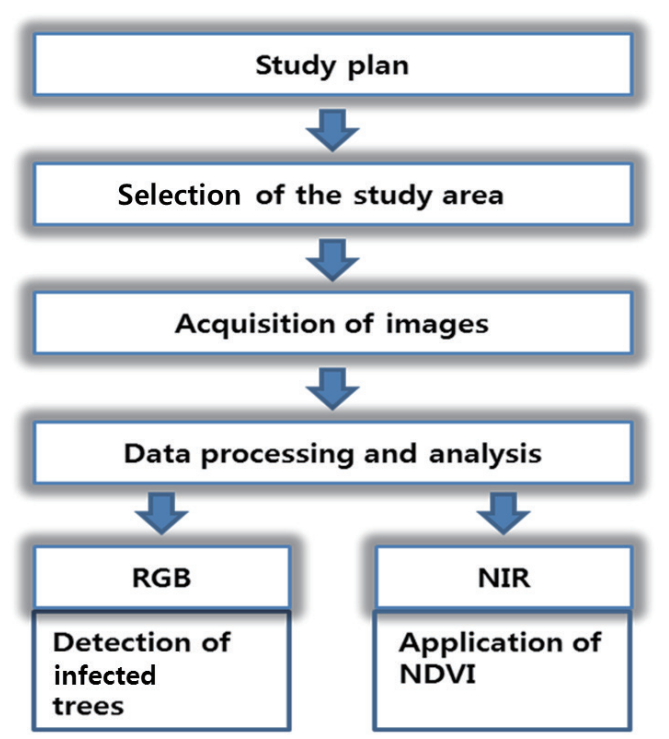

Fig. 2. Study flow. 


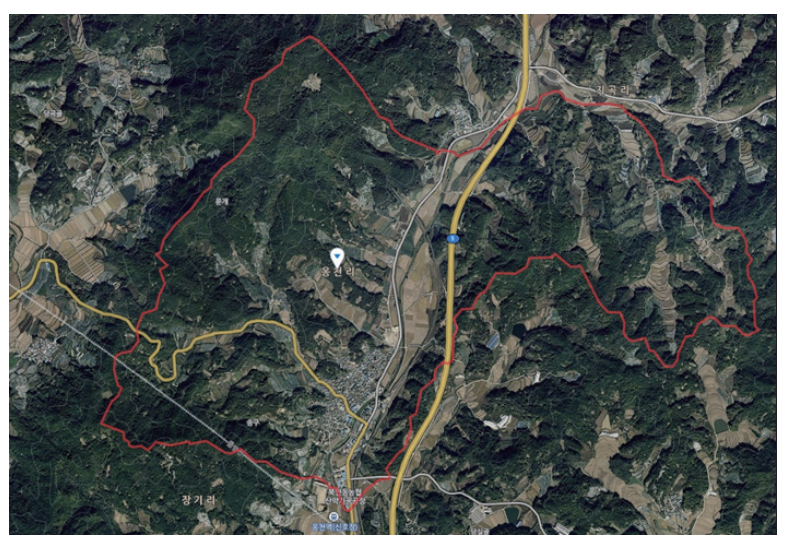

Table 1

Specifications of UAV.

\begin{tabular}{lc}
\hline Item & Description \\
\hline Type & Fixed wing \\
Weight & $2.5 \mathrm{~kg}$ \\
Wingspan & $1 \mathrm{~m}$ \\
Height above take-off location & $75-750 \mathrm{~m}$ \\
Flight duration & $50 \mathrm{~min}$ \\
Cruise speed & $80 \mathrm{~km} / \mathrm{h}$ \\
Camera & $\mathrm{Nex}-5 \mathrm{R}(16.1 \mathrm{MP})$ \\
Resolution & $2.4-24 \mathrm{~cm}$ \\
\hline
\end{tabular}

Fig. 3. (Color online) Study area.

type Exmore APS HD CMOS enabled taking photographs with high sensitivity and low noise. The radio antenna could transmit UAV speed, altitude, coordinates, and other flight data to a radio modem of the ground controller by wireless communication. The pitot tube measured the difference in pressure between the opening of the front and that of the side to monitor the speed and altitude of the UAV is placed in an eBox.

The area for taking images using UX5 was about $4.76 \mathrm{~km}^{2}$. The RGB and NIR images were extracted from the aerial images obtained, and these were converted to RGB and NIR ortho images by data processing. Table 1 shows the specifications of the UAV. ${ }^{(12)}$

\section{Data Processing}

The aerial images were postprocessed by the Trimble Business Center (TBC). The number of aerial images was 2626 and those of the RGB and NIR images were 1308 each. The numbers of RGB and NIR images differed from that of aerial images because the angle of view of the cameras are different. The degree of overlap of aerial images was $80 \%$, and ground sample distance (GSD) of the ortho image was $10 \mathrm{~cm}$. Aerial images were imported to a data processing module along with their locations, orientations, and camera calibrations. Geometric errors in the raw images from the UAV were significant owing to the dynamic platform from which they were captured and the imprecise UAV position and orientation sensors. To correct for errors in the positions and orientations of the aerial images, we used photogrammetric methods to adjust the photo stations. This is carried out in the data processing module first as an adjustment with tie points. This module automatically finds tie points in all available stations using state-of-the-art computer vision algorithms, and then the TBC adjusts the stations simultaneously for a best fit. Automatically matched photo tie points are distributed densely over the complete project, even in a challenging low-texture terrain.

The ortho images processed from the RGB and NIR images and the RGB images were used for the detection of infected trees, and the NIR images were used for calculating the normalized difference vegetation index (NDVI), which was necessary for the early detection of infected trees. Figure 4 shows the forest geospatial data. 


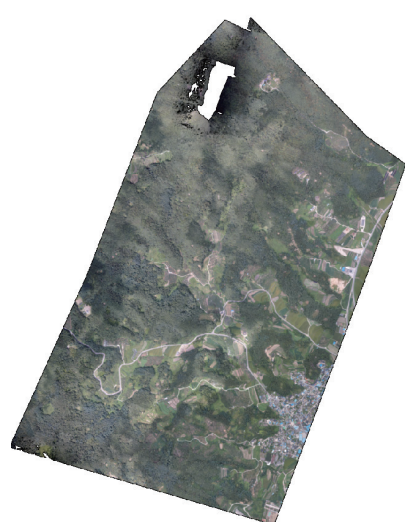

(a)

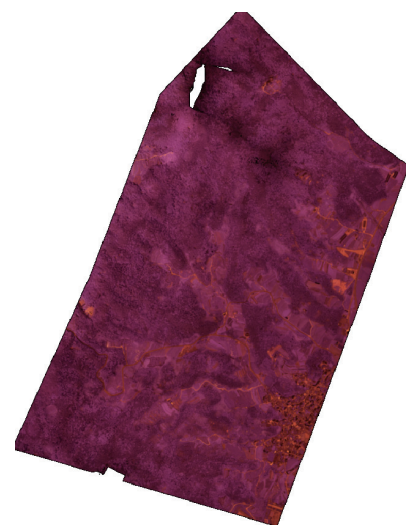

(b)

Fig. 4. (Color online) Forest geospatial data. (a) RGB ortho image and (b) NIR ortho image.

\section{Detection of Infected Trees and Application of NDVI}

\subsection{Detection of infected trees}

The location of infected trees was indicated by the RGB ortho image used for the detection. The infected trees appear brown, and we identified the location and quantity of these trees using the RGB ortho images. Information on the position of the infected trees can be used as basic data for the management of diseases, whereas information on the quantity of infected trees was accurate and could contribute to the preparation of a forest management plan. Figure 5 shows the information on the infected trees and Table 2 shows the coordinates of the infected trees.

Even though the target area was subjected to pest control, some infected trees remained and were detected as shown in Fig. 5. Pest control should be carried out in conjunction with field investigation to prevent the spread of BURSXY.

\subsection{Application of NDVI in extracting infected trees}

In area $\mathrm{A}$, even though BURSXY was not the cause of death of some trees, many trees suspected of being infected were detected. NDVI was calculated from the NIR images of the target area using the band math of the NIR camera. Table 3 and Fig. 6 show the bandwidth of the Nex-5R camera.

NDVI indicates plant "greenness" or photosynthetic activity and is one of the most commonly used vegetation indices. ${ }^{(1)}$ Vegetation indices are based on the observation that different surfaces reflect light of different wavelengths. Photosynthetically active vegetation, in particular, absorbs most of the red light that hits it while reflecting much of the NIR light. Vegetation that is dead or stressed reflects more red light and less NIR light. Likewise, nonvegetative surfaces have a much more even reflectance across the light spectrum. Figure 7 shows the NDVI of area A. 


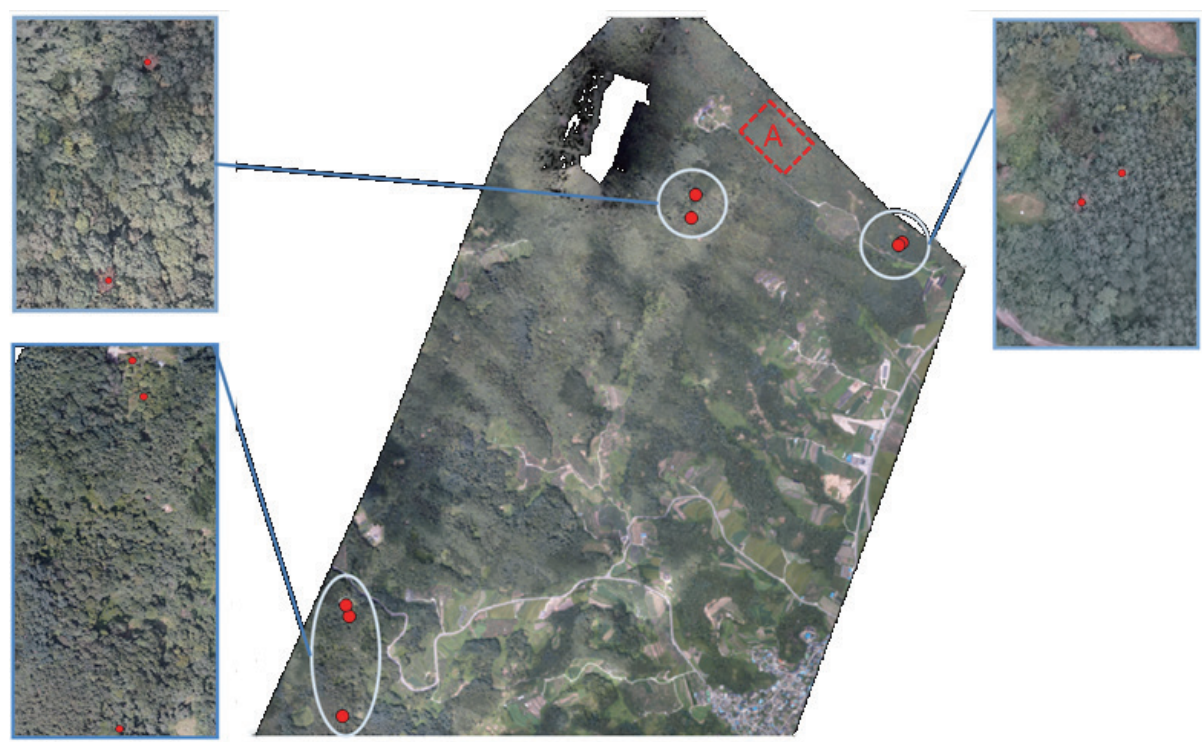

Fig. 5. (Color online) Information on infected trees.

Table 2

Coordinates of infected trees.

\begin{tabular}{lcc}
\hline No. & Northing $(\mathrm{m})$ & Easting $(\mathrm{m})$ \\
\hline 1 & 454244.32 & 171859.73 \\
2 & 454571.81 & 171880.31 \\
3 & 454605.74 & 171871.22 \\
4 & 455876.12 & 173001.03 \\
5 & 455950.73 & 173013.92 \\
6 & 455785.43 & 173678.83 \\
7 & 455795.32 & 173689.51 \\
\hline
\end{tabular}

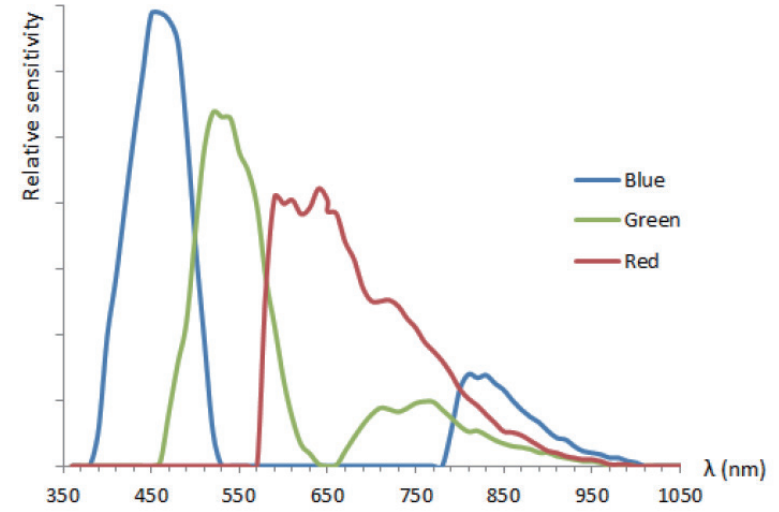

Fig. 6. (Color online) Relative sensitivity of the band.
Table 3

Bandwidth of Nex-5R camera.

\begin{tabular}{lc}
\hline Band & Value \\
\hline 1 (Red) & $570-970 \mathrm{~nm}, 50 \%$ thresholds \\
& at $590-790 \mathrm{~nm}$ \\
\hline 2 (Blue) & $460-640$ and $660-970 \mathrm{~nm}$, \\
& $50 \%$ thresholds at $490-590 \mathrm{~nm}$ \\
\hline & $390-530$ and $780-1020 \mathrm{~nm}$, \\
3 (Green) & $50 \%$ thresholds in the blue region \\
& are $420-500 \mathrm{~nm}$ and \\
& in the NIR region $790-890 \mathrm{~nm}$ \\
\hline
\end{tabular}

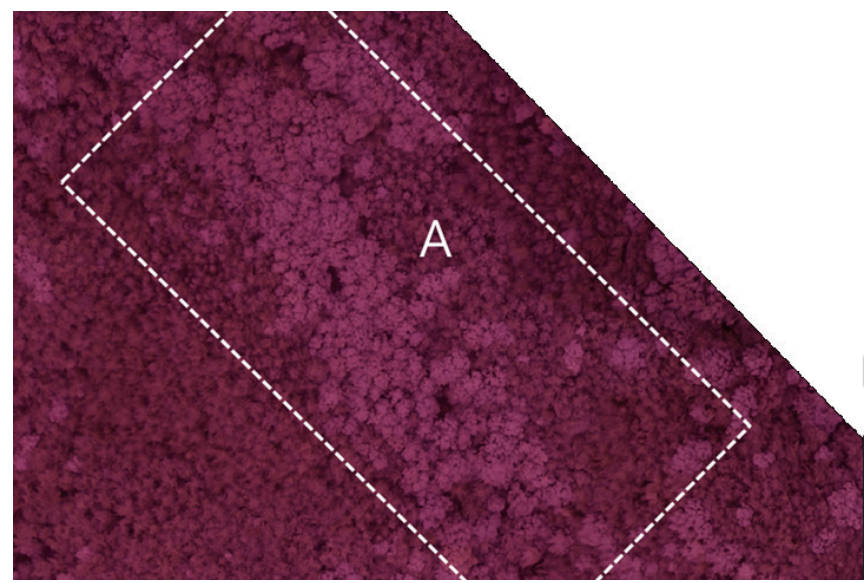

Fig. 7. (Color online) NDVI of area A. 
The NDVI of area A was relatively lower than that of a normal forest. This research shows that BURSXY was detected effectively by our method. Furthermore, in the case of the detection of infected trees using ortho images, the coordinate information is important, that is, if this is accompanied by GPS, a marked improvement of the effectiveness of pest control measures is expected. With NDVI, the judgment of dying trees, which were hardly detectable using RGB images, was possible. Further research about the prehension of the characteristics of normal trees and BURSXY infected trees will be helpful for the management of the disease caused by BURSXY.

\section{Conclusions}

In this research, UAV images were used to detect infected trees effectively. RGB and NIR images were captured and processed to produce ortho images. We conclude that with RGB ortho images, the effective detection of infected trees was possible. Because the target area was subjected to pest control measures, there were not many infected trees but some that remained infected were detected. Because the coordinate information can be easily acquired using the ortho images, GPS will be helpful in determining the position of infected trees. By this method, we expect that the efficiency and accuracy of the work can be greatly improved as compared with the conventional manpower forest survey. In addition, some trees suspected of being infected but were not actually infected, were found on the basis of the calculated NDVI of the target area. The NDVI of such an area was lower than that of an area with non-infected trees. Field investigation of the area seems to be necessary, and further research about the difference in characteristics between BURSXY-infected trees and non-infected trees will be beneficial for improving the management of the disease caused by BURSXY. The results of this study are expected to support big data for forest management.

\section{Acknowledgments}

This research was supported by Basic Science Research Program through the National Research Foundation of Korea(NRF) funded by the Ministry of Science and ICT (No. NRF2018R1C1B6004021).

\section{References}

1 D. Y. Han, J. B. Park, and J. W. Huh: J. Korean Soc. Surv. Geod. Photogramm. Cartography 36 (2018) 451. https://doi.org/10.7848/ksgpc.2018.36.6.451

2 K. R. Lee, J. H. Seong, Y. K. Han, and W. H. Lee: J. Korean Soc. Surv. Geod. Photogramm. Cartography 37 (2019) 9. https://doi.org/10.7848/ksgpc.2019.37.1.9

3 L. Goparaju and F. Ahmad: Spatial Inf. Res. 4 (2019) 1. https://doi.org/10.1007/s41324-019-00261-5

4 J. H. Oh, Y. J. Jang, and C. N. Lee: J. Korean Soc. Surv. Geod. Photogramm. Cartography 36 (2018) 565. https://doi.org/10.7848/ksgpc.2018.36.6.565

5 J. H. Kim and J. H. Kim: J. Korean Soc. Surv. Geod. Photogramm. Cartography 36 (2018) 223-233. https://doi. org/10.7848/ksgpc.2018.36.4.223

6 J. H. Seong, Y. K. Han, and W. H. Lee: J. Korean Soc. Surv. Geod. Photogramm. Cartography 36 (2018) 279. https://doi.org/10.7848/ksgpc.2018.36.4.279 
7 G. Shukla, R. D. Garg, P, Kumar, H. S. Srivastava, and P. K. Garg: Spatial Information Research 26 (2018) 573. https://doi.org/10.1007/s41324-018-0200-4

8 K. B. Kang, H. S. Kim, J. W. Jwa, H. C. Kim, and M. J. Kang: J. Korean Academia-Industrial Cooperation Soc. 18 (2017) 117. https://doi.org/10.5762/KAIS.2017.18.10.117

9 Korea Forest Service: http: www.forest.go.kr/ (accessed April 2019).

10 J. K. Park and K. Y. Jung: Korean Academia-Industrial Cooperation Soc. 19 (2018) 602. https://doi. org/10.5762/KAIS.2018.19.2.602

11 S. H. Jung and H. S. Kim: J. Korean Convergence Soc. 8 (2017) 9. https://doi.org/10.15207/JKCS.2017.8.6.009

12 Trimble Inc.: http:www.trimble.com (accessed April 2019).

\section{About the Authors}

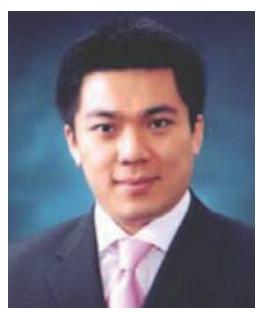

Kap Yong Jung received his B.S., M.S., and Ph.D. degrees in civil engineering from Chungnam National University, Korea in 2003, 2009, and 2013, respectively. His research interests are in the areas of geodetic science, surveying, and geospatial information. (jungjusa@hanmail.net)

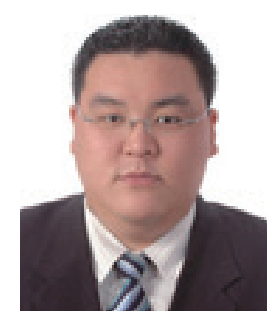

Joon Kyu Park is currently a professor in the Department of Civil Engineering at Seoil University. He received his B.S., M.S., and Ph.D. degrees in civil engineering from Chungnam National University, Korea in 2001, 2003, and 2008, respectively. His research interests are in the areas of GPS and geo-spatial information engineering. (jkpark@seoil.ac.kr) 